\title{
Prognostic factors affecting survival and recurrence in gastric carcinoma
}

\author{
Ahmed Itaimi*, Oussema Baraket, Wissem Triki, Karim Ayed and Sami Bouchoucha \\ Université tunis el manar. Faculté de medicine de tunis, Tunisia
}

\begin{abstract}
Background: Gastric adenocarcinoma is a serious and frequent digestive cancer. It is the second leading cause of cancer deaths in the world after colorectal cancer. The bad prognosis is caused by several factors. The identification of prognostic factors will make it possible to better codify therapeutic management in order to improve survival and reduce recurrence rates.
\end{abstract}

Aim: Identify the main prognostic factors affecting overall survival and recurrence in gastric adenocarcinoma.

Methods: Retrospective study involving patients undergoing surgery for gastric adenocarcinoma between January 2006 and March 2016 in Habib Bougatfa Hospital in Tunisia.

Results: Overall survival was $58 \%$ at 3 years. Survival without recurrence was 20 months. Survival without recurrence at 1 year, 3 years and 5 years were respectively $88 \%, 73 \%$ and $70 \%$. Eleven patients had a recurrence. The median time to onset of recurrence was 12 months. Of the 11 patients who had a recurrence, nine had died. Mortality due to recurrence was $82 \%$. In univariate analysis, overall survival and recurrence were influenced by several factors. After multivariate analysis, lymph node invasion, occurrence of recurrence, number of lymph nodes removed less than 15 , presence of signet-ring cell carcinoma and tumor stage 3 and 4 significantly influenced survival. Independent factors influenced recurrences were: the presence of lymph nodes and signet ring cell carcinoma.

Conclusion: Several factors influence overall survival and recurrence in gastric adenocarcinoma. The lymph node invasion and the anatomo-pathological factors are the most important. Improving therapeutic outcomes requires early diagnosis and careful surgery.

Abbreviations: LBVI: Lymphatic and/or blood vessel invasion; NLR: Neutrophil lymphocytes ratio.

\section{Introduction}

Gastric carcinoma is a serious cancer and a public health problem around the world. Despite the decrease in the global incidence of this cancer [1], it remains the fifth most common type of cancer [2]. It has a poor prognosis and it is the second leading cause of digestive cancer death in the world after colorectal cancer [1]. Survival at 5 years does not exceed $20 \%$ for all stages combined. The curative treatment of gastric adenocarcinoma is based on surgical excision with lymph node dissection. In addition, the contribution of chemotherapy and radiotherapy is considerable in improving the prognosis. The prognosis of gastric cancer depends on several factors. The identification of these factors makes it possible to classify patients according to the prognostic risk, and to better codify the management and the therapeutic protocols. This will lead to improve survival and reduce recurrence rates. The objective of this study is to identify the main prognostic factors affecting survival and recurrence of gastric adenocarcinoma.

\section{Patients and methods}

Retrospective study involving 60 patients undergoing surgery for gastric adenocarcinoma in the General Surgery Department of Habib Bougatfa Hospital, Bizerte, from January 2012 to March 2016.

\section{Patients}

It included: Patients undergoing surgery for gastric adenocarcinoma and having gastric resection during the study period.
It was not included: Patients who were not operated (metastatic or unresectable cancer), adenocarcinoma of the cardia, and other histological forms (lymphoma, endocrine tumors, stromal tumors ...).

Evaluation criteria in the study were the overall survival, and factors conditioning the occurrence of recurrence and disease-free survival.

\section{Data collection}

Data was collected from patient records. We were interested in epidemiological, clinical, operative and pathological data. The degree of parietal invasion and lymph node extension was established according to the 7th edition of the TNM classification of the International Union Against Cancer (UICC) published in 2010 [3]. D1,5 dissection is defined as a D2 dissection without spleno-pancreatectomy. The diagnosis of recurrence was only made in the presence of obvious histological or radiological evidence.

*Correspondence to: Ahmed Itaimi, Université tunis el manar. Faculté de medicine de tunis, Belvedere City, Monia Building, Apt 230, Bizerte7000, Tunisia, Tel: 21620100835; E-mail: ahmed.itaimi@hotmail.com

Key words: gastric cancer, prognosis

Received: October 20, 2018; Accepted: November 12, 2018; Published: November 15, 2018 


\section{Statistic study}

The prognosis was studied in two parts:

The overall survival was analyzed from the whole series and the event that changed the curve was death.

The recurrence was analyzed from the group of patients undergoing curative surgery and not died postoperatively. survival without recurrence was considered and the event that modifies the curve was recurrence.

Data was analyzed using SPSS software version 19.0. The search for prognostic factors for survival and recurrence was performed in univariate analysis by comparing the curves by the Log rank test and then by multivariate analysis in Cox regression method. Multivariate analysis allowed the calculation of adjusted relative risks, measuring the proper role of each factor. In all statistical tests, the significance level was set at 0.05 .

\section{Results}

We assessed 60 patients. The median age was 60 years old. There was a male predominance with a sex ratio $(\mathrm{M} / \mathrm{F})$ of 2.52 . The average time to diagnosis was 7 months. Perioperatively, the lesion was located in the gastric antrum in almost half of cases (47\%). Locoregional invasion was observed in 5 cases and the tumor was metastatic in 4 cases. Subtotal gastrectomy was performed in $47 \%$ of cases and total gastrectomy in $53 \%$ of cases. An enlarged resection was performed in $8 \%$ of cases. D1.5 lymph node dissection was performed in almost half the cases followed by $\mathrm{D} 2$ dissection in $35 \%$ of cases. Resection was curative (R0) in $93 \%$ of cases.

At the histological study, the average tumor size was $4 \mathrm{~cm}$. Adenocarcinoma was well differentiated in $15 \%$ of cases, moderately differentiated in $50 \%$ of cases and undifferentiated in $35 \%$ of cases. The tumor was classified T1-T2 in 10 cases and T3-T4 (advanced tumor) in 50 cases. Lymph node invasion was observed in $70 \%$ of cases. The median number of lymph nodes removed was 14 . Overall survival was $58 \%$ at 3 years (Figure 1). The average survival was 22 months. In univariate analysis, the factors that influenced overall survival were: ratio of invaded lymph node/removed lymph node $>10 \%$, tumor stage 3 and 4 , the occurrence of recurrence, presence of lymph nodes in perioperative, signet-ring cell carcinoma, and lymph node invasion. In multivariate analysis, the presence of signet-ring cell carcinoma (Figure 2), lymph node invasion (Figure 3), tumor stage 3 and 4 (Figure 4), the number of lymph nodes removed $<15$ (Figure 5), and the occurrence of recurrence (Figure 6) decreased survival significantly (Table 1). The median survival without recurrence was 20 months. Disease-free survival at 1 year, 3 years and 5 years were respectively $88.6 \%, 73.3 \%$

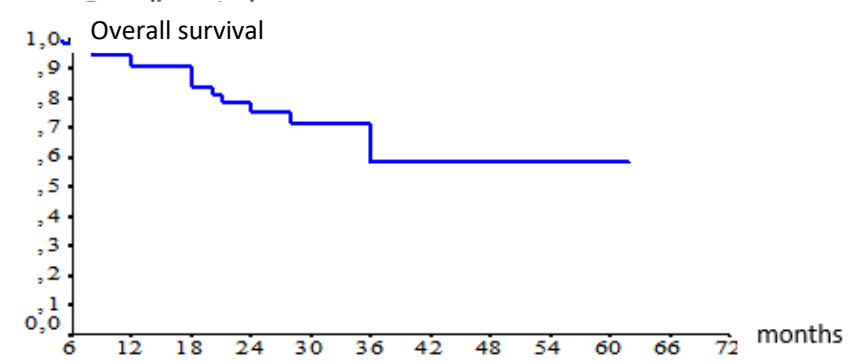

Figure 1. Overall survival according Kaplan Meier method

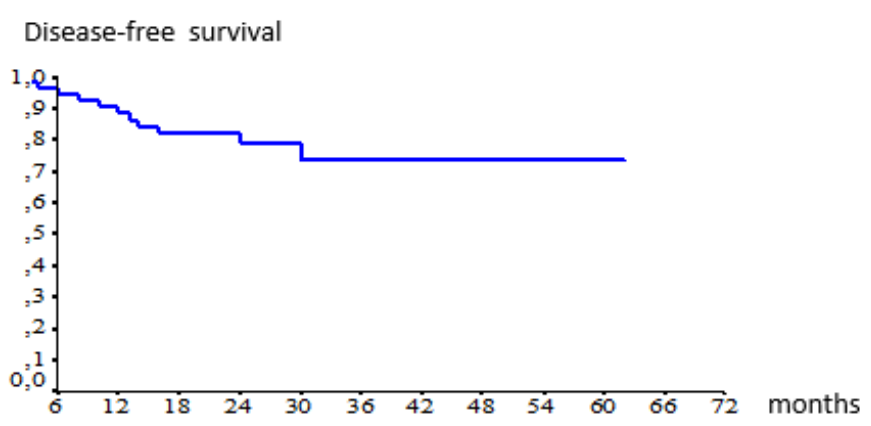

Figure 2. Overall survival according the presence of signet-ring cell

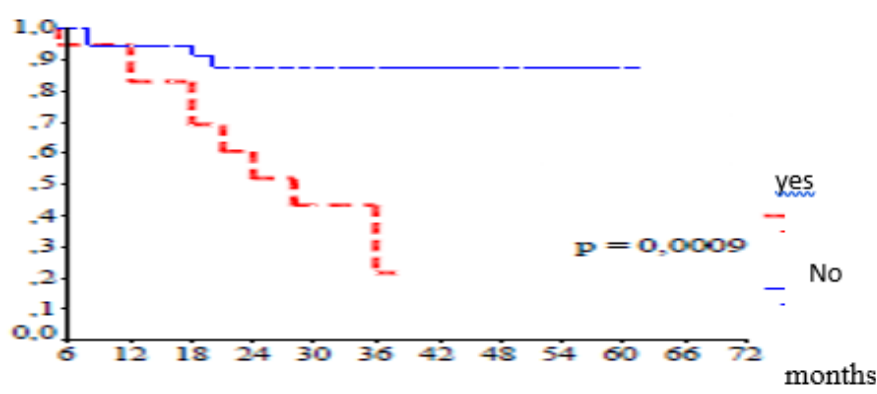

Figure 3. Overall survival according lymph node invasion

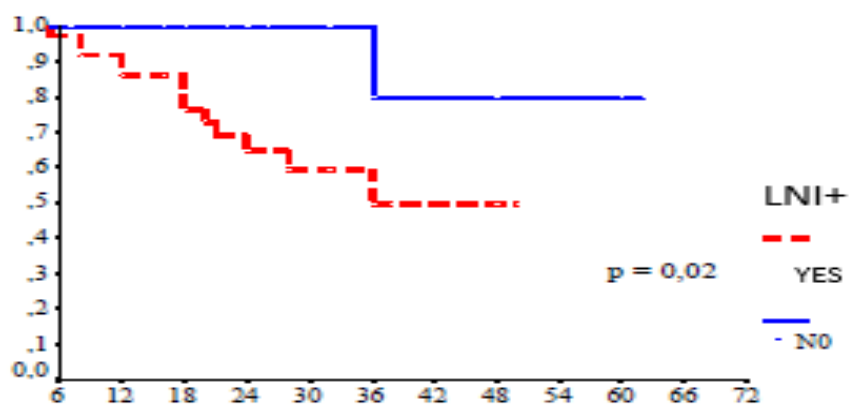

Figure 4. Overall survival according tumor stage

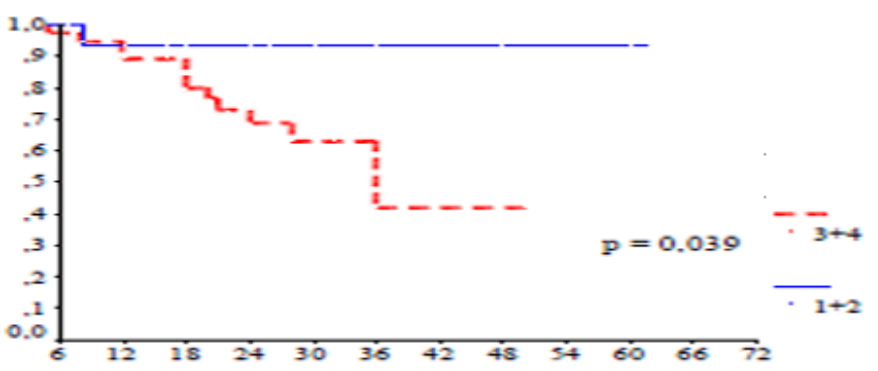

Figure 5. Overall survival according number of lymph node removed

and $70 \%$ (Figure 7). Eleven patients had recurrence (18\% of cases). The median time to onset of recurrence was 12 months. Of the 11 patients who had a recurrence, 9 patients died. The factors predictive of recurrence in univariate analysis were: the presence of lymph nodes in peroperatively, signet-ring cell carcinoma, gastric linitis, the ratio invaded nodes/removed nodes $>10 \%$, the tumor differentiation and the realization of total gastrectomy. After multivariate analysis independent factors that increased the risk of recurrence were: the presence of lymph nodes in peroperatively and signet-ring cell carcinoma (Table 2). 


\section{Discussion}

Gastric adenocarcinoma is a cancer with a poor prognosis. This poor prognosis depends on several factors that can be classified into patient-related factors, others related to the tumor and those related to surgery. The analysis of the different prognostic factors makes it possible to adapt the therapeutic management in order to improve the results in terms of survival and recurrence. A review of literature showed that 5-year survival of resected gastric adenocarcinoma ranged from $26-60 \%$, 10-year survival ranged from $26.3-34.2 \%$, and median survival is about $23.2 \%$ at 48 months [4]. Our results in terms of overall survival approach the results of the literature.

Regarding prognostic factors related to the patient, the majority of authors agree that epidemiological factors (age, sex) do not influence the prognosis of gastric adenocarcinoma $[5,6]$, and in our study neither survival nor recurrence were influenced by these factors. The presence of a palpable abdominal mass at the clinical examination of the patient reflects the evolved nature of the tumor and is considered a poor

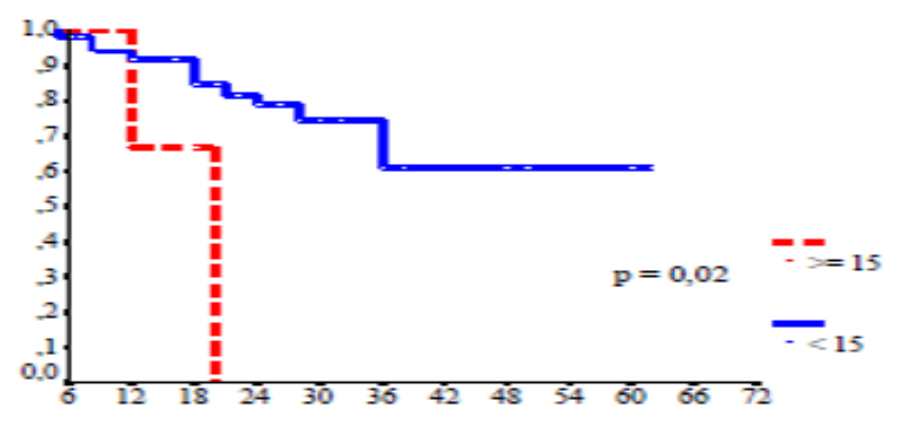

Figure 6. Overall survival according the occurrence of recurrence

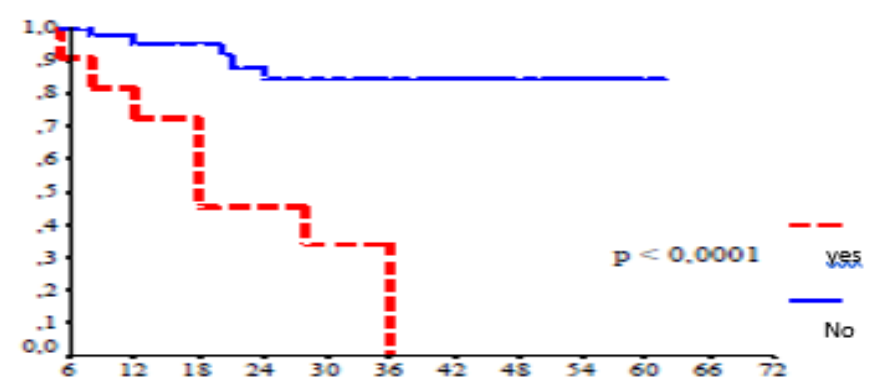

Figure 7. Disease-free survival according Kaplan Meier method

Table 1. Independent factors influenced overall survival in multivariate analysis

\begin{tabular}{|c|c|c|c|}
\hline Variables & $\boldsymbol{p}$ & $\begin{array}{c}\text { Adjusted } \\
\text { Hazard Ratio }\end{array}$ & $\begin{array}{c}\text { Confidence } \\
\text { Interval (CI) }\end{array}$ \\
\hline Lymphatic invasion & 0.023 & 4 & $0.4-38.4$ \\
\hline Tumor stage 3 and 4 & 0.039 & 1 & $0.1-10.4$ \\
\hline $\begin{array}{c}\text { Number of lymph } \\
\text { nodes removed }<15\end{array}$ & 0.041 & 1.5 & $0.4-5.3$ \\
\hline Signet-ring cell & 0.024 & 2.3 & $0.6-9$ \\
\hline Occurrence of recurrence & 0.017 & 4.6 & $1.3-16.1$ \\
\hline
\end{tabular}

Table 2. Independent factors of recurrence in multivariate analysis

\begin{tabular}{|c|c|c|c|}
\hline Variables & $\boldsymbol{p}$ & $\begin{array}{c}\text { Adjusted } \\
\text { Hazard ratio }\end{array}$ & $\begin{array}{c}\text { Confidence } \\
\text { Interval (CI) }\end{array}$ \\
\hline $\begin{array}{c}\text { Signet ring-cell } \\
\text { carcinoma }\end{array}$ & 0.039 & 5.2 & $1.1-25.1$ \\
\hline $\begin{array}{c}\text { Presence of lymph } \\
\text { nodes }\end{array}$ & 0.03 & 4.3 & $1.1-16.7$ \\
\hline
\end{tabular}

prognostic factor affecting survival. Eight percent of our patients had an abdominal mass on examination and its presence did not affect the prognosis. This could be explained by the fact that the presence of a mass was not necessarily synonymous with the advanced stage of the tumor. Some studies showed an association between intraoperative transfusions and poor long-term prognosis. The authors explained this correlation by the non-specific immunosuppression produced by transfused blood. A meta-analysis [7] including 16 studies showed that the 5-year survival rate was decreased for transfused patients $(\mathrm{RR}=0.74,95 \%$ confidence interval $[\mathrm{CI}]=0.69-0.79)$, with increased rates of recurrence and postoperative complications. Furthermore, the preoperative serum albumin level not only reflects the nutritional status of the patient but was also considered as a prognostic factor in gastric adenocarcinoma [4,8] and the more this hypo albuminemia is important, the less the survival is better. Also, the neutrophil lymphocytes ratio (NLR) was analyzed by several studies as a prognostic factor. A meta-analysis [9] concluded that high NLR reflects both a heightened neutrophil-dependent inflammatory response and a decreased lymphocyte mediated antitumor immune reaction, which may weaken the lymphocyte-mediated anti-tumor cellular immune response and contribute to aggressive tumor biology, cancer progression and poor prognosis.

Regarding the location of the tumor, the distal location was observed in half of the cases, and the survival was not influenced by the location of the tumor. A meta-analysis with a systematic review of the literature [10] including 50 studies concluded that localization in the upper third of the stomach was associated with a poor prognosis $(p<0.001)$, and after excluding tumors from the gastroesophageal junction, the prognosis was bad for the tumors of the cardia. Several authors also identified tumor size as a predictor of survival and proposed to include tumor size in the UICC TNM classification to improve survival prediction in patients undergoing curative resection. A recent study [11] showed that tumor size was a critical factor in the indication of adjuvant chemotherapy for T3-4aN0M0 tumors. This study concluded that tumor size was an independent prognostic factor. In addition, adjuvant chemotherapy improved the prognosis in patients with T3-4aN0M0 gastric cancer with tumors greater than $5 \mathrm{~cm}$, whereas these results were not found for tumors smaller than 5 $\mathrm{cm}$. Although this study had its limitations; retrospective study with small sample size, further trials are needed to validate its findings. In this study tumor size had not influenced overall survival. Regarding the histological type, several histological classification systems for gastric cancers were described and there is still controversial as to which classification gives a better prognostic correlation. The World Health Organization (WHO) classification published in 2010 appears to be the most detailed of all histological classification systems and is considered an independent prognostic factor [12]. Most authors attributed a poor prognosis to signet-ring cell carcinoma compared to other histological types. The presence of signet-ring cell had significantly influenced the overall survival of our patients ( $\mathrm{p}=0.0009$ ) (Figure 2). In addition, the undifferentiated forms and the mucinous type had a poor prognosis compared to the papillary and tubular types [12]. The presence of lymphatic and/or blood vessel invasion (LBVI) is considered as an independent factor of poor prognosis for N0 tumors and lymph node recurrence is more common in LBVI + tumors [13]. The same study showed that patients with pT3N0 tumor or patients with N0 tumor who had lymph node dissection less than D2 can benefit from adjuvant chemotherapy if histopathological examination found LBVI, and 
according to authors, patients who were initially treated by endoscopic resection and in whom LBVI were identified on the resection specimen, these patients should be referred secondarily to surgery for gastrectomy with complementary lymph node dissection. The degree of parietal invasion also significantly influenced the prognosis of gastric adenocarcinoma. Overall, survival is better for poorly evolved tumors (T1, T2) compared to advanced tumors (T3, T4) [14]. Lymph node (N) status is recognized as the most important factor conditioning the prognosis of gastric adenocarcinoma especially for untreated forms of cancer $[15,16]$. Indeed, the overall survival rate increases from 90 $70 \%$ respectively in the absence and in the presence of lymph node metastases [16]. Lymph node invasion was observed in $70 \%$ of our patients and significantly reduced survival $(\mathrm{p}=0.02)$ (Figure 3 ).

The objective of surgery in gastric adenocarcinoma is to obtain a curative excision of the gastric lesion (R0) with adequate lymph node dissection. In our study, curative resection was performed in 56 patients (93\%). The 5-year survival was $60 \%$ in case of curative resection (R0) and $0 \%$ in case of palliative resection leaving a macroscopic tumor residue $(\mathrm{p}<0.001)$. The curative resection is considered as an important factor of prognosis $[17,18]$. The extent of lymph node dissection was a matter of debate. The benefit of extensive dissection (D2 or more) remains controversial. Western surgeons did not find any benefit from extensive dissection in the improvement of survival, indeed the results of the Dutch and British randomized studies, did not show a gain of overall survival after D2 dissection compared to the D1 dissection, the expenses postoperative mortality rates much higher $[19,20]$. Contrary to this point of view, Japanese surgeons demonstrated that better locoregional control by extensive dissection results in improved survival by preventing locoregional recurrences and thus reducing distant metastases. There were a few randomized controlled trials, published over the past two decades, evaluating the impact of different types of ganglion dissection on survival.

\section{Conclusion}

The prognosis of gastric adenocarcinoma remains poor. Curative treatment is based on surgical excision with lymph node dissection. The overall prognosis of gastric adenocarcinoma remains bleak. In our series as in the literature, there are several factors influencing the prognosis. Lymph node invasion and anatomo-pathological factors were the most important.

\section{References}

1. Ferro A, Peleteiro B, Malvezzi M, Bosetti C, Bertuccio P, et al. (2014) Worldwide trends in gastric cancer mortality (1980-2011), with predictions to 2015, and incidence by subtype. Eur J Cancer 50: 1330-1344. [Crossref]

2. Ferlay J, Soerjomataram I, Dikshit R, Eser S, Mathers C, et al. (2015) Cancer incidence and mortality worldwide: sources, methods and major patterns in GLOBOCAN 2012. Int J Cancer 136: 359-367. [Crossref]

3. Edge SB, Compton CC (2010) The American Joint Committee on Cancer: the 7th edition of the AJCC cancer staging manual and the future of TNM. Ann Surg Oncol 17: 1471-1474. [Crossref]

4. Onate Ocana LF, Aiello Crocifoglio V, Gallardo Rincon D (2007) Serum albumin as a significant prognostic factor for patients with gastric carcinoma. Ann Surg Oncol 14: 381-389. [Crossref]

5. Nakamura R, Saikawa Y, Takahashi T, Takeuchi H, Asanuma H, et al. (2011) Retrospective analysis of prognostic outcome of gastric cancer in young patients. Int $J$ Clin Oncol 16: 328-334. [Crossref]

6. Bautista M, Jiang SF, Armstrong M (2014) Impact of age on clinicopathological features and survival of patients with non-cardia gastric adenocarcinoma. $J$ Gastric Cancer 4: 238-246. [Crossref]
7. Li L, Zhu D, Chen X, Huang Y, Ouyang M, et al. (2015) Perioperative allogenenic blood transfusion is associated with worse clinical outcome for patients undergoing gastric carcinoma surgery. A meta-analysis. Medicine (Baltimore) 94: 1574-1580.

8. Lien YC, Hsieh CC, Wu YC (2004) Preoperative serum albumin level is a prognostic indicator for adenocarcinoma of the gastric cardia. J Gastrointest Surg 8: 1041-1048. [Crossref]

9. Zhang X, Zhang W, Feng LJ (2014) Prognostic significance of neutrophil lymphocyte ratio in patients with gastric cancer: a meta-analysis. PLoS One 9: e111906. [Crossref]

10. Petrelli F, Ghidini M, Barni S, Steccanella F, Sgroi G (2017) Prognostic role of primary tumor location in non-metastatic gastric cancer: a systematic review and meta-analysis of 50 studies. Ann Surg Oncol 24: 2655-2664. [Crossref]

11. Chen S, Ou-Yang LY, Nie RC, Li YF, Xiang J (2017) Tumor size is a critical factor in adjuvant chemotherapy for T3-4an0m0 gastric cancer patients after D2 gastrectomy. Gastroenterol Res Pract 2017: 4928736. [Crossref]

12. Zheng HC, Zheng YS, Xia P, Xu XY, Xing YN, et al. (2010) The pathobiological behaviors and prognosis associated with Japanese gastric adenocarcinomas of pure WHO histological subtypes. Histol Histopathol 25: 445-452. [Crossref]

13. Lee JH, Kim MG, Jung MS, Kwon SJ (2015) Prognostic significance of lymphovascular invasion in node-negative gastric cancer. World J Surg 39: 732-739. [Crossref]

14. Saito H, Osaki T, Murakami D, Sakamoto T, Kanaji S, et al. (2006) Macroscopic tumo size as a simple prognostic indicator in patients with gastric cancer. Am J Surg 192: 296-300. [Crossref]

15. Kim JP, Lee JH, Kim SJ (1998) Clinicopathologic characteristics and prognostic factors in 10783 patients with gastric cancer. Gastric Cancer 1: 125-129.

16. Chen R, He Q, Cui J, Bian S, Chen L (2014) Lymph node metastasis in early gastric cancer. Chin Med J (Engl) 127: 560-567. [Crossref]

17. Kunisaki C, Shimada H, Ono HA, Otsuka Y, Matsuda G, et al. (2006) Comparison of results of surgery in the upper third and more distal stomach. $J$ Gastrointest Surg 10: 718-726. [Crossref]

18. Sierra A, Regueira FM, Hernandez-Lizoain JL (2003) Role of the extended lymphadenectomy in gastric cancer surgery: experience in a single institution. Ann Surg Oncol 10: 219-226. [Crossref]

19. Bonenkamp JJ, Hermans J, Sasako M, van de Velde CJ, Welvaart K, et al. (1999) Extended lymph-node dissection for gastric cancer. $N$ Engl J Med 340: 908-914. [Crossref]

20. Cuschieri A, Weeden S, Fielding J (1999) Patient survival after D1 and D2 resections for gastric cancer: long-term results of the MRC randomized surgical trial. Surgical co-operative group. Br J Cancer 79: 1522-1530. [Crossref]

Copyright: (C2018 Itaimi A. This is an open-access article distributed under the terms of the Creative Commons Attribution License, which permits unrestricted use, distribution, and reproduction in any medium, provided the original author and source are credited. 